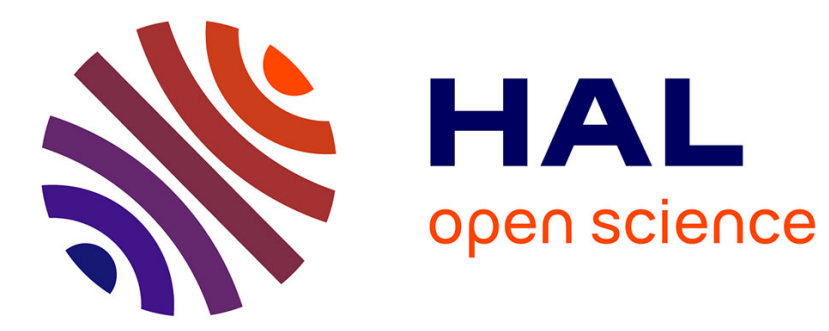

\title{
Interfacial intermixing in InAs/GaSb short-period-superlattices grown by molecular beam epitaxy
}

E. Luna, B. Satpati, J. B. Rodriguez, A. N. Baranov, E. Tournié, A. Trampert

\section{- To cite this version:}

E. Luna, B. Satpati, J. B. Rodriguez, A. N. Baranov, E. Tournié, et al.. Interfacial intermixing in InAs/GaSb short-period-superlattices grown by molecular beam epitaxy. Applied Physics Letters, 2010, 96 (2), 10.1063/1.3291666 . hal-01618582

\section{HAL Id: hal-01618582 \\ https://hal.science/hal-01618582}

Submitted on 31 May 2021

HAL is a multi-disciplinary open access archive for the deposit and dissemination of scientific research documents, whether they are published or not. The documents may come from teaching and research institutions in France or abroad, or from public or private research centers.
L'archive ouverte pluridisciplinaire HAL, est destinée au dépôt et à la diffusion de documents scientifiques de niveau recherche, publiés ou non, émanant des établissements d'enseignement et de recherche français ou étrangers, des laboratoires publics ou privés. 


\title{
Interfacial intermixing in InAs/GaSb short-period-superlattices grown by molecular beam epitaxy
}

\author{
E. Luna, ${ }^{1, a)}$ B. Satpati, ${ }^{1, b)}$ J. B. Rodriguez, ${ }^{2}$ A. N. Baranov, ${ }^{2}$ E. Tournié, ${ }^{2}$ and A. Trampert ${ }^{1}$ \\ ${ }^{1}$ Paul-Drude-Institut für Festkörperelektronik, Hausvogteiplatz, 5-7, D-10117 Berlin, Germany \\ ${ }^{2}$ Université Montpellier 2, CNRS, UMR 5214, IES, CC 067, Place Eugène Bataillon, F-34095 Montpellier \\ cedex 5, France
}

(Received 30 October 2009; accepted 18 December 2009; published online 12 January 2010)

\begin{abstract}
The unique properties of the noncommon-atom InAs/GaSb short-period-superlattices (SPSL) strongly depend on the interface structure. These interfaces are characterized using transmission electron microscopy (TEM). The compositional sharpness is obtained from the comparison of the experimental contrast in $\mathbf{g}_{002}$ two-beam dark-field TEM images with simulated intensity profiles, which are calculated assuming that the element distribution profiles are described by sigmoidal functions. The interfacial intermixing, defined by the chemical width, is obtained for SPSL with different periods and layer thicknesses, even in the extreme case of nominally less than 3 ML thick InAs layers. Nominal 1 ML InSb layers intentionally inserted are also identified. () 2010 American Institute of Physics. [doi:10.1063/1.3291666]
\end{abstract}

During many years InAs/GaSb superlattices (SLs) have been the subject of investigation due to their unique potential device applications. ${ }^{1,2}$ In each case, device performance is a strong function of the quality of the interfaces. Using different techniques, it was found that the interface InAs-on-GaSb is extended while the interface GaSb-on-InAs is more abrupt. $^{3-6}$ This arises from the exchange reactions between As and $\mathrm{Sb}^{7}$ as well as the incorporation of $\mathrm{Sb}$ into InAs promoted by the strong segregation tendency of $\mathrm{Sb}^{8}{ }^{8}$ While most of the studies focus on the interfacial roughness (i.e., steps and islands), little is known about the interfacial intermixing (i.e., composition profile). Despite the importance in quantifying the interface stoichiometry, the determination of the interfacial intermixing in this noncommon-atom (NCA) system still proves to be a challenging task because only a few experimental techniques can be used. Using transmission electron microscopy (TEM) techniques, we propose an innovative and straightforward method for a reliable and systematic characterization of these interfaces. The method is of general applicability and allows for the detection of variations in the interface width that are smaller than the resolution of the 002 dark-field (DF) TEM imaging (about $0.5 \mathrm{~nm})$.

We illustrate the potential of our method by showing case studies of samples with extremely different degrees of interfacial intermixing. The samples consist of InAs/GaSbbased short-period-superlattices (SPSLs) and were grown by molecular beam epitaxy (MBE) at growth temperatures $T_{\mathrm{g}}$ $=390-420{ }^{\circ} \mathrm{C}$ using controlled shutter sequences and specific soaking procedures at the interfaces. Cross-sectional TEM foils were prepared in the $[110]$ and $[\overline{1} 10]$ projections, using mechanical thinning followed by Ar-ion milling. The TEM investigation was carried out using a Jeol JEM 3010 microscope operating at $300 \mathrm{kV}$, equipped with a GATAN slow-scan charge-coupled device camera.

\footnotetext{
${ }^{\text {a)} E l e c t r o n i c ~ m a i l: ~ l u n a @ p d i-b e r l i n . d e . ~}$

${ }^{b}$ Present address: Centre for Advanced Material Processing, Central Mechanical Engineering Research Institute, Mahatma Gandhi Avenue, Durgapur-713209, India.
}

High-resolution transmission electron microscopy (HRTEM) images (not shown here) reveal atomically smooth interfaces giving an interfacial roughness of only one monolayer (ML). In principle, a lattice distortion analysis of the HRTEM micrographs would be possible detecting the local strain (in unit cell dimensions) and thus identifying the different strained layers. Further strain analysis would determine the change in lattice parameter across the interfaces, i.e., the composition profiles. However, in the present case, due to the small amount of strain involved (the lattice mismatch between InAs and $\mathrm{GaSb}$ is $\sim 0.6 \%$ ), this method only allows for a rough visualization of the different layers but not for an accurate determination of the strain and, subsequently, of the composition profile across the interface. An alternative approach is then required. Mahalingam et al. ${ }^{6}$ determined the change in composition across a $17 \mathrm{ML}$ InAs/6 ML $\mathrm{In}_{0.25} \mathrm{Ga}_{0.75} \mathrm{Sb} \mathrm{SL}$ structure using sophisticated reconstruction of the exit wave function in HRTEM. More recently, preliminary composition profiles from $15 \mathrm{ML}$ InAs/17 ML GaSb SLs obtained using atom probe tomography have been presented ${ }^{10}$ where complicated data reconstruction analysis is essential for a correct interpretation of the results. In contrast, our proposal to evaluate the interfacial intermixing is based on conventional TEM techniques and, although a dedicated data analysis is required, all the steps are straightforward to implement.

In particular, the method relies on the analysis of twobeam DF-TEM images obtained with the diffraction vector $\mathbf{g}=002$, which is sensitive to the chemical composition for semiconductors with zinc blende structure. The idea is based on the proposal of a distribution profile for the different constituent elements that we put into the calculation of the corresponding diffracted intensity under kinematic conditions $\left(\mathrm{I}_{002}\right)$. We compare the simulated intensity $\left(\mathrm{I}_{002}\right)$ with the experimental one $\left(\mathrm{I}_{002}^{\mathrm{exp}}\right)$ and look for the composition profiles best fitting the experimental image. The procedure would resemble the analysis of $\mathrm{x}$-ray diffraction data where the layer information (composition, thickness, strain, etc.) is extracted after comparing the experimental and simulated curves. The key issue is the identification of the element 

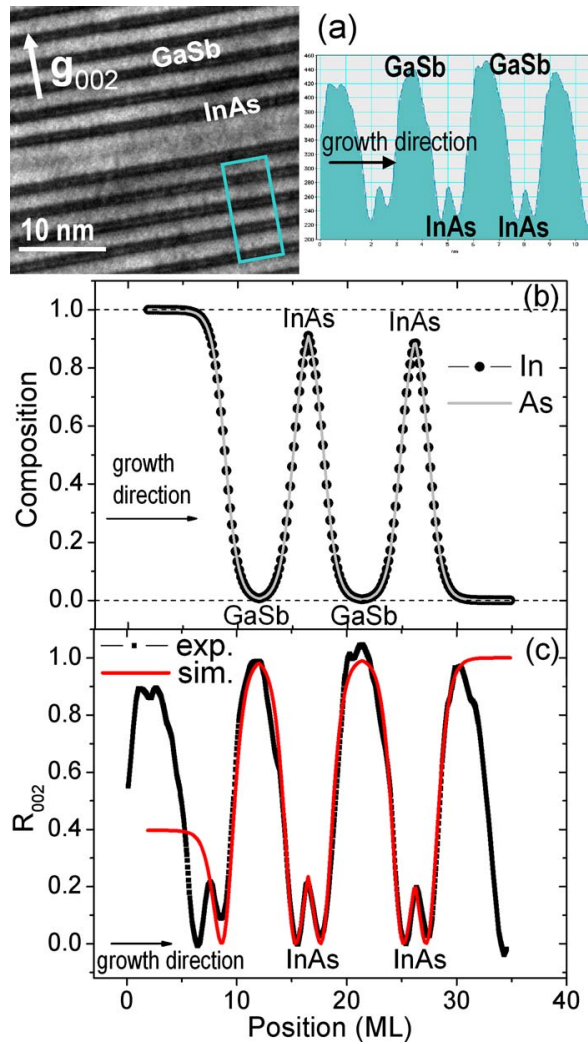

FIG. 1. (Color online) (a) $\mathbf{g}_{002}$ DF TEM micrograph of a (3 ML InAs/6 ML $\mathrm{GaSb}$ ) SPSL, together with an intensity linescan. (b) Proposed element distribution profiles yielding to the simulated $\mathrm{R}_{002}$ best reproducing the experimental intensity ratio $(c)$.

distribution profile. From the analysis of directly determined experimental composition profiles in III-arsenides and dilute nitride heterostructures, we have already demonstrated that the smooth variation of the element concentration with the position at the interface follows a sigmoidal law, with the interface width, $L$, as the main fitting parameter. ${ }^{11,12}$ Such functional dependence for the transition region arises from the kinetics of the interface formation during MBE growth ${ }^{13}$ and hence can be considered as a universal behavior regardless of the semiconductor system considered. Thus, the realistic distributions for In and As in the InAs layer of the NCA $\mathrm{SL}$ (those for $\mathrm{Ga}$ and $\mathrm{Sb}$ are obtained after mass conservation, $[\mathrm{In}]+[\mathrm{Ga}]=100 \%$ and $[\mathrm{As}]+[\mathrm{Sb}]=100 \%)$ are obtained assuming that the change in composition across the two interfaces of the thin buried layer follows the sigmoidal law described in Ref. 12.

Figure 1(a) shows a 002 DF-TEM micrograph of a (3 ML InAs/6 ML GaSb) SPSL together with an intensity linescan. In order to avoid measuring absolute intensities, the scattered intensity of the layers is normalized to that of $\mathrm{GaSb}$ of the same thickness, $\mathrm{R}_{002}=\mathrm{I}_{002}^{\text {layer }} / \mathrm{I}_{002}^{\mathrm{GaSb}}$. Since the identity of cation and anion both change across the heterointerface, we have to input the contributions of the III and V-element sublattices, separately. Figure 1(b) represents the composition profiles best reproducing the experimental data [Fig. 1(c)]. As observed, the agreement between the simulation and the experiment is remarkable, in particular at the two minima defining the interfaces (i.e., dark lines in the 002 DF-TEM image) stemming from the functional dependence of $\mathrm{I}_{002}^{(\mathrm{In}, \mathrm{Ga}) \text { As }}$ on the In concentration. ${ }^{14}$

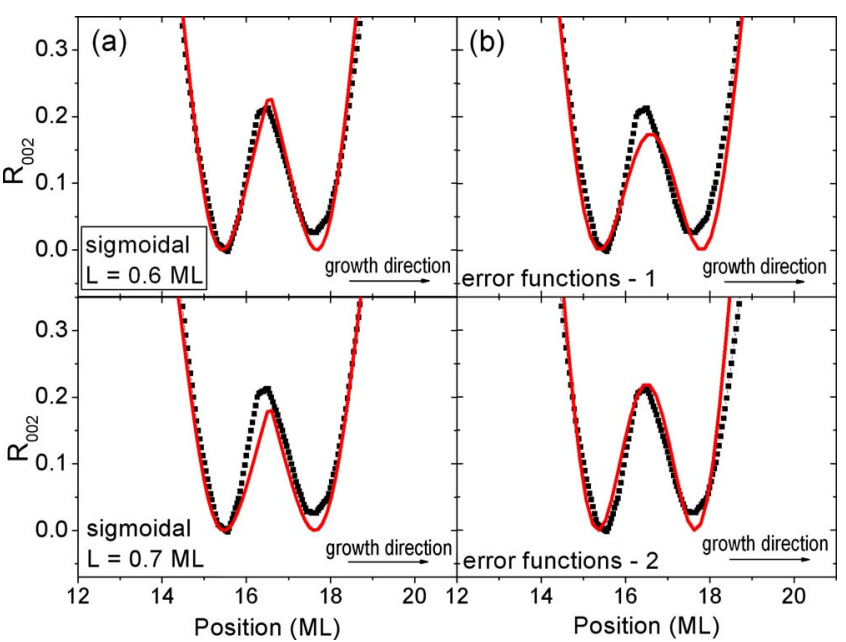

FIG. 2. (Color online) Magnification of the interface-related features in the intensity profile displaying (a) the sensitivity of the method to variations in the interface width as small as $0.1 \mathrm{ML}$, and (b) the results assuming element profiles based on error functions.

This sample exhibits abrupt interfaces for both III and V-element sublattices, $L_{\mathrm{II}}=L_{\mathrm{V}}=0.6 \mathrm{ML}$, i.e., the chemical intermixing is very low. The In and As contents inside the InAs layer are smaller than $100 \%$, i.e., the layer is composed of $(\mathrm{In}, \mathrm{Ga})(\mathrm{As}, \mathrm{Sb})$, because of (i) the thin $3 \mathrm{ML}$ InAs layers and (ii) the finite compositional transition at the interfaces. Moreover, because of the small layer thickness involved (3 ML), the interface intermixing is significant and accountable for the nonachievement of the nominal In content before the next GaSb layer comes. In order to estimate the plausible presence of segregation effects, we consider input distribution profiles that are obtained from the combination of a segregated profile derived after Muraki's phenomenological model $^{15}$ and the sigmoidal function for the description of the interface. ${ }^{11,16}$ Segregation of Sb would have a large impact on the shape of the $\mathrm{I}_{002}$ minimum (i.e., dark line) defining the InAs-on-GaSb interface, which will get broader and asymmetric as the segregation efficiency, $R$, increases. The appearance of the symmetric $\mathrm{I}_{002}^{\mathrm{GaSb}}$ profile would also be affected. All in all, if we take segregation into account and try to fit the experimental data, we find that $R_{\mathrm{Sb}}<0.5$, i.e., $\mathrm{Sb}$ segregation is negligible. We end up in a similar result $\left(R_{\mathrm{In}}\right.$ $<0.4$ ) if we consider In segregation into GaSb (Ref. 17) instead. Finally, the method described here is sensitive to variations of $L \pm 0.15 \mathrm{ML}$, as variations in the interface width as small as $0.1 \mathrm{ML}$ are clearly discernible from the comparison of the simulated and experimental $\mathrm{R}_{002}$. The differences are mainly observed at the interface-related features of the contrast profile as it is shown in the zoomed plot of Fig. 2(a).

Note that composition profiles based on other functions rather than the sigmoidal can be input into the calculation as well, for example, those based on error-functions, the otherwise widely accepted functional dependence at interfaces. Interestingly, the use of distribution profiles based on error functions yields to noticeable disagreements between $R_{002}$ and $\mathrm{R}_{002}^{\exp }$, as it is shown in Fig. 2(b) that displays two of the "best" fittings using error functions. Therefore, it can be inferred that the InAs/GaSb interfaces are more properly described by the sigmoidal function. 


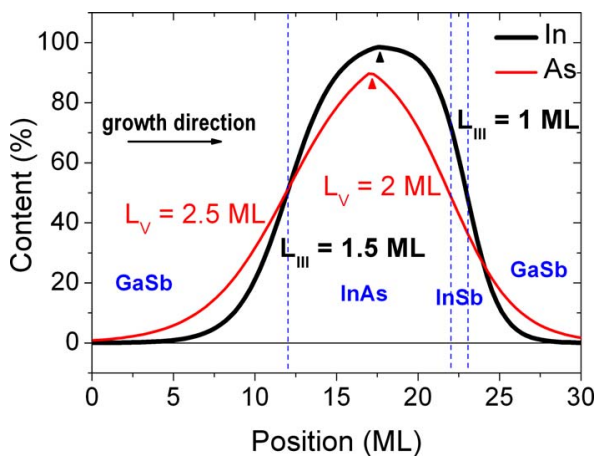

FIG. 3. (Color online) Composition profiles across a (10 ML GaSb/10 ML InAs/1 ML InSb) SPSL showing (i) chemically broad interfaces and (ii) a different intermixing at the III- and V-element sublattice. The small shift in the distribution profiles for In and As is considered as an indirect evidence of the presence of the $1 \mathrm{ML}$ InSb layer (arrows: indicate points at which the interface profiles are joined).

It is known that different growth conditions can affect the interface properties. That is the case, for instance, of the following (10 ML GaSb/10 ML InAs/1 ML InSb) SPSL. The intermediate $1 \mathrm{ML}$ InSb is introduced for controlling the interface character. ${ }^{9}$ The corresponding In and As composition profiles that agree best with the simulated $\mathrm{I}_{002}$ are displayed in Fig. 3. Contrary to the previous case, here we find chemically broad interfaces, with $L_{\mathrm{V}}$ as large as $2.5 \mathrm{ML}$ and a different intermixing of the III and V-element sublattice, $L_{\mathrm{III}} \neq L_{\mathrm{V}}$, with $L_{\mathrm{III}}=1-1.5 \mathrm{ML}$, while $L_{\mathrm{V}}=2-2.5 \mathrm{ML}$. It should be reminded that the method allows for the independent determination of the interface width in the III and V-element sublattice, as the As and In distribution profiles are separately input into the calculation. In this sample, we find that the interface InAs-on-GaSb is broader than the interface of GaSb-on-In(Sb,As). These results agree with those reported by Mahalingam et al..$^{6}$ and correlate with the growth conditions for this sample, in particular an As-flux soaking at the interface, although it could also be explained in relation to the segregation tendency of $\mathrm{Sb}^{8}{ }^{8}$ The small shift in the distribution profiles for In and As could be considered as an indirect evidence of the presence of the intentionally inserted $1 \mathrm{ML}$ InSb layer. Indeed, the detection of the individual nominal $1 \mathrm{ML}$ InSb is quite ambitious for conventional DFTEM. We find however that the experimental contrast profiles of all investigated $\mathbf{g}_{002}$ images for this sample cannot be explained without including the $1 \mathrm{ML}$ InSb layer. Moreover, only assuming an intermixed layer composed of $(\mathrm{In}, \mathrm{Ga})(\mathrm{S}$ $\mathrm{b}, \mathrm{As}$ ), instead of pure $\mathrm{InSb}$, produces a matching between the simulated and experimental data.

Finally, Fig. 4(a) represents a highly magnified $\mathbf{g}_{002}$ DFTEM micrograph of a few ML thick SPSL, composed of stacks of nominally (6 ML GaSb/2 ML InAs/1.5 ML InSb/2 ML InAs). The darker stripes correspond to the InAs/InSb trilayers, and the high morphological perfection of the SPSL is clearly visible. Again, the detection of the individual layers is quite ambitious. Nevertheless, it is possible to find tiny contrast variations in the intensity profile [Fig. 4(b)], indicating an InAs/InSb/InAs layer sequence. We simulate the contrast intensity with the element profiles depicted in Fig. 4(c), assuming an interface width of only $L=0.5$ ML for both the
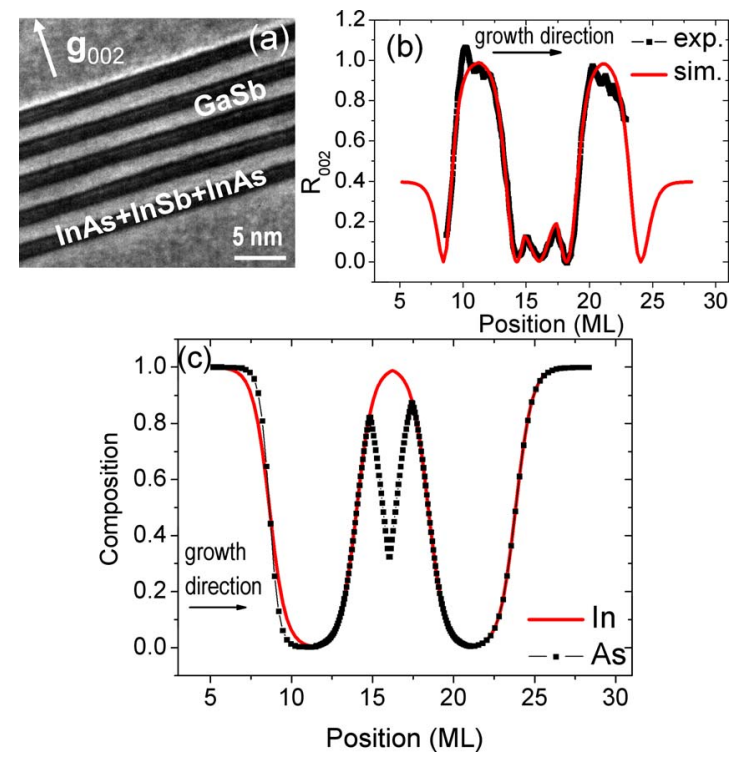

FIG. 4. (Color online) (a) $\mathbf{g}_{002}$ DF TEM image of the few ML thick (6 ML $\mathrm{GaSb} / 2 \mathrm{ML}$ InAs/1.5 ML InSb/2 ML InAs) SPSL, (b) simulated intensity profile that agrees best with the experimental results obtained after the composition profiles shown in (c).

III and V-element sublattices. The agreement between the simulation and the experiment is remarkable and all the individual layers are identified.

We acknowledge B. Jenichen for a careful reading of the manuscript and A. Pfeiffer for technical assistance. Part of this work has been sponsored by the European Commission (Project No. FP6-017383, DOMINO).

${ }^{1}$ H. Kroemer, Physica E (Amsterdam) 20, 196 (2004).

${ }^{2}$ M. Razeghi, Eur. Phys. J.: Appl. Phys. 23, 149 (2003).

${ }^{3}$ M. W. Wang, D. A. Collins, T. C. McGill, R. W. Grant, and R. M. Feenstra, J. Vac. Sci. Technol. B 13, 1689 (1995).

${ }^{4}$ R. Kaspi, J. Cryst. Growth 201, 864 (1999).

${ }^{5}$ R. M. Feenstra, D. A. Collins, D. Z.-Y. Ting, M. W. Wang, and T. C. McGill, Phys. Rev. Lett. 72, 2749 (1994).

${ }^{6}$ K. Mahalingam, K. G. Eyink, G. J. Brown, D. L. Dorsey, C. F. Kisielowski, and A. Thust, Appl. Phys. Lett. 88, 091904 (2006).

${ }^{7}$ Q. Xie, J. E. Van Nostrand, J. L. Brown, and C. E. Stutz, J. Appl. Phys. 86, 329 (1999).

${ }^{8}$ J. Steinshnider, J. Harper, M. Weimer, C.-H. Lin, S. S. Pei, and D. H. Chow, Phys. Rev. Lett. 85, 4562 (2000).

${ }^{9}$ J. B. Rodriguez, P. Christol, L. Cerutti, F. Chevrier, and A. Joullié, J. Cryst. Growth 274, 6 (2005).

${ }^{10}$ M. Müller, A. Cerezo, and G. D. W. Smith, Microscopy of Semiconducting Materials Conference (MSM XVI, Oxford, 2009).

${ }^{11}$ E. Luna, F. Ishikawa, P. D. Batista, and A. Trampert, Appl. Phys. Lett. 92, 141913 (2008).

${ }^{12}$ E. Luna, F. Ishikawa, B. Satpati, J. B. Rodriguez, E. Tournié, and A. Trampert, J. Cryst. Growth 311, 1739 (2009).

${ }^{13}$ E. Luna, A. Guzmán, and A. Trampert, 26th North-American MBE conference, Princeton, 2009 (unpublished).

${ }^{14}$ H. Cerva, Appl. Surf. Sci. 50, 19 (1991).

${ }^{15}$ K. Muraki, S. Fukatsu, Y. Shiraki, and R. Ito, Appl. Phys. Lett. 61, 557 (1992).

${ }^{16}$ The profiles predicted by the segregation models are based on the assumption of initial perfectly squarelike interfaces, which is unlikely to exist in reality.

${ }^{17}$ C. Renard, X. Marcadet, J. Massies, I. Prévot, R. Bisaro, and P. Galtier, J. Cryst. Growth 259, 69 (2003). 